\title{
Combination therapy in idiopathic pulmonary fibrosis: the way ahead will be hard
}

\author{
Athol Wells \\ Affiliation: Interstitial Lung Disease Unit, Royal Brompton Hospital, London, UK. \\ Correspondence: Interstitial Lung Disease Unit, Royal Brompton Hospital, Sydney St, Chelsea, London, \\ SW3 6NP, UK. E-mail: rbhildarnht.nhs.uk
}

@ERSpublications

Combination therapy is the future for IPF treatment but many questions remain regarding tolerability and study design http://ow.ly/Kuykl

In retrospect, 2014 will be seen as the year in which idiopathic pulmonary fibrosis (IPF) therapies came of age. Treatment effects reported previously were inconclusive with regard to both pirfenidone (two positive studies [1,2] and a negative study [2]) and nintedanib (a single phase II study in which trends were marginal due to under-powering [3]). The ASCEND pirfenidone trial [4] and the two INPULSIS nintedanib trials [5] ended uncertainty. The two agents appear remarkably similar in both tolerability and efficacy, with a 50\% reduction in forced vital capacity (FVC) decline. Whither now? No biomarker data exist to indicate that either agent might be more efficacious in individual patients. Initially, the routine use of these therapies is likely to be sequential, with one agent replaced by the other when side-effects are problematic or treatment failure is evident, however this is defined. In the longer term, combinations of pirfenidone and nintedanib appear attractive in principle, based both on the possibility of synergy in a disease in which there is a multiplicity of coactivated pathways, and also on precedence in other lung diseases including asthma, chronic obstructive pulmonary disease, lung cancer and pulmonary artery hypertension [6]. However, the road to combination regimens in IPF is likely to have many twists and turns. Two particular problems need to be addressed, even supposing that additive or synergistic benefits exist (something that has yet to be established). Tolerability issues are important, as both agents give rise to significant gastrointestinal side-effects. It should also be understood from the outset that demonstrating efficacy will not be straightforward. Intense multinational activity was needed to show treatment benefits against placebo arms characterised by FVC declines approximating $220 \mathrm{~mL} \cdot \mathrm{year}^{-1}$. The treatment advances of 2014 sound the death knell of placebo-controlled evaluation in IPF: it seems inevitable that combination regimens will be compared with monotherapy. The rate of FVC decline of approximately $110 \mathrm{~mL} \cdot \mathrm{year}^{-1}$ in patients receiving either pirfenidone or nintedanib will pose major challenges with regard to powering future trials [6]. The current issue of the European Respiratory Journal (ERJ) features two studies that are highly relevant to tolerability and study design issues.

OguRA et al. [7] report a randomised, double-blind, phase II, dose escalation trial in 50 Japanese IPF patients, in which the safety, tolerability and pharmacokinetics of nintedanib were assessed, alone and when added to pirfenidone. Studied interventions consisted of placebo $(\mathrm{n}=12)$, nintedanib $50 \mathrm{mg}$ twice daily for 14 days $(\mathrm{n}=6)$, nintedanib $100 \mathrm{mg}$ twice daily for 14 days $(\mathrm{n}=8)$ or nintedanib $150 \mathrm{mg}$ twice daily for 28 days $(n=24)$. As placebo nintedanib was given to only 12 patients, including six not taking pirfenidone, the greater interest lies in the comparison between patients receiving nintedanib alone $(n=17)$ and those in whom nintedanib was added to pirfenidone $(n=21)$. No severe adverse events were observed. Mild or moderate events were seen in nine out of 17 patients treated with nintedanib alone and in 10 out of 21 patients treated with combination therapy. The most common adverse events were gastrointestinal, exclusively present in the full dose arm and occurring more frequently with combination therapy (11 episodes in 13 patients) than with nintedanib alone (four episodes in 11 patients). The

Received: March 182015 | Accepted: March 182015

Conflict of interest: Disclosures can be found alongside the online version of this article at erj.ersjournals.com

Copyright (CERS 2015 
pharmacokinetics of pirfenidone were not influenced by the addition of nintedanib. By contrast, there was a trend toward lower nintedanib exposure, as judged by maximum plasma concentration and area under the curve at steady state, when nintedanib was added to pirfenidone.

The only clear conclusion to emerge from the study, albeit in a tiny patient cohort, was that combination therapy was not associated with serious adverse events. The absence of major safety concerns in a small group of patients needed to be established in a preliminary evaluation of this sort in order to allow more definitive observations to be accumulated in further studies. The tolerability and pharmacokinetic data were both highly inconclusive. Whilst the overall prevalence of adverse events differed little between the two treatment groups, the larger number of gastrointestinal episodes seen with combination therapy may or may not be indicative of future problems. The duration of treatment was short. Furthermore, combination therapy was studied only in patients who had tolerated pirfenidone, although this bias may be less important than it seems: presumably, if combination therapy becomes a future standard of care, agents will be introduced sequentially with escalation to a combination regimen only if an initial treatment is tolerated. Finally, the pharmacokinetic data may have been heavily influenced by outliers, always a risk when studies are seriously under-powered. The value of the study of Ogura et al. [7] seems to lie primarily in its unanswered questions, which, it can be hoped, will give structure to future work.

Also in the current issue of the ERJ, LEY et al. [8] have attempted to improve mortality prediction in IPF. The GAP (Gender-Age-Physiology) model had previously been validated as a baseline-risk prediction model for mortality in IPF [9]. However, as known by all clinicians and enshrined by a disease behaviour classification, endorsed for use in unclassifiable interstitial lung disease [10], baseline prognostic data are often trumped by observed short-term longitudinal change. Combining baseline and longitudinal data is logical and reflects thinking in clinical practice. The study population was definitive in its size $(n=1109)$ and was made up of the placebo arms of two pirfenidone trials [2] and all randomised subjects in a trial of interferon- $\gamma$ [11]. Baseline GAP status (considered separately as the GAP calculator and the GAP index) and changes in a number of variables during the first 6 months of the trial period were examined against subsequent mortality. Vital status was known in all patients during the second 6 months of the trial periods but follow-up was necessarily less complete thereafter.

The data are complex but the major interest in the study lies in the accuracy of the final composite models, defined by sophisticated model performance statistics. The authors propose a user-friendly approach in which GAP predictors are combined with a history of respiratory hospitalisation and 24-week change in FVC. As is so often the case, the devil lies in the detail. In table 4 of the manuscript, it becomes clear that there is very little to choose between a number of combinations of variables, with regard to prognostic evaluation. GAP status was accurate when combined with permutations of respiratory hospitalisation, 24-week FVC change, 24-week change in the 6-min walking distance, and the University of California San Diego dyspnoea scale (which was more accurate as a baseline than as a serial change variable). An earlier risk prediction model, developed in this cohort by DU BoIs et al. [12], provided broadly similar prognostic guidance.

The authors argue for the use of the model in clinical practice in individual patients, but this recommendation can be questioned. Extrapolation from average population data to individual patients is a chancy business. Respiratory hospitalisations are necessarily defined nonspecifically in clinical trials but have value because they capture acute exacerbations. In individual patients, the clinician can be far more precise, assigning a poor prognosis at the time of diagnosing an acute exacerbation, rather than depending upon a history of nonspecific respiratory hospitalisation in the previous 6 months. Other serial variables may be reasonably reliable on average but in individual patients it may be obvious that changes are unreliable, especially when due to confounding by comorbidities (which are under-represented in clinical trials but are the warp and weft of clinical practice in IPF). The process of defining disease progression does not always work well by formula but requires review and reconciliation of all available data. Serial changes in gas transfer and gas exchange are not included in the presented models: although less useful, on average, in a trial cohort of mild to moderate severity, large changes in these variables are of cardinal importance in individual patients. The findings of LEY et al. [8] are helpful in so far as they focus the attention of clinicians on key variables but, in the end, prognostic evaluation in clinical practice requires an individualised rather than a formulaic approach.

The greater relevance of the current study would seem to lie in the need to refine trial end-points when combination therapies are studied against monotherapy. As discussed earlier, the continued use of serial FVC as a stand-alone primary end-point is likely to lead to insuperable difficulties in powering studies to demonstrate efficacy. A composite approach is likely to be needed and the findings of LEY et al. [8] provide a springboard to inform this vitally important task. It is worth closing with an important caveat. The validation of composite end-points against subsequent mortality is plainly important but we should not 
take this to mean that an end-point, validated in this way, is a surrogate for mortality, especially when mortality is relatively short term, as in the current study. In trials of advanced small cell and nonsmall cell lung cancer, progression-free survival correlates well with mortality within trial periods $[13,14]$ but, with one or two exceptions, is not an accurate predictor of longer term mortality in malignant disease in general [15]. The development of accurate composite end-points for future trial work may require that the components are justified as much by logic as by arcane statistical analysis. Composite end-points can be rationalised in two classes: 1) "bad news" composites, e.g. mortality, respiratory hospitalisation, major decline in FVC or the 6-min walking distance, or the development of pulmonary hypertension; and 2) chronic disease progression composites, in which FVC change is amalgamated with changes in measures of gas transfer, symptoms and computed tomography variables. The former may give rise to more accurate short-term prediction, but chronic progression composite end-points may have more relevance to long-term outcome. Serial variables should not be grouped simply because they are user-friendly but on the basis of a logical discourse, with practicability taken into account.

Combination therapy is the IPF treatment of the future. It is evident from data presented in the current issue of the ERJ that many questions remain to be answered with regard to tolerability and future study design.

\section{References}

1 Taniguchi H, Ebina M, Kondoh Y, et al. Pirfenidone in idiopathic pulmonary fibrosis. Eur Respir J 2010; 35 : 821-829.

2 Noble PW, Albera C, Bradford WZ, et al. Pirfenidone in patients with idiopathic pulmonary fibrosis (CAPACITY): two randomised trials. Lancet 2011; 377: 1760-1769.

3 Richeldi L, Costabel U, Selman M, et al. Efficacy of a tyrosine kinase inhibitor in idiopathic pulmonary fibrosis. N Engl J Med 2011; 365: 1079-1087.

4 King TE Jr, Bradford WZ, Castro-Bernardini S, et al. A phase 3 trial of pirfenidone in patients with idiopathic pulmonary fibrosis. N Engl J Med 2014; 370: 2083-2092.

5 Richeldi L, du Bois RM, Raghu G, et al. Efficacy and safety of nintedanib in idiopathic pulmonary fibrosis. $N$ Engl J Med 2014; 370: 2071-2082.

6 Wuyts WA, Antoniou KM, Borensztajn K, et al. Combination therapy: the future of management for idiopathic pulmonary fibrosis? Lancet Respir Med 2014; 2: 933-942.

7 Ogura T, Taniguchi H, Azuma A, et al. Safety and pharmacokinetics of nintedanib and pirfenidone in idiopathic pulmonary fibrosis. Eur Respir J 2015; 45: 1382-1392.

8 Ley B, Bradford WZ, Weycker D, et al. Unified baseline and longitudinal mortality prediction in idiopathic pulmonary fibrosis. Eur Respir J 2015; 45: 1374-1381.

9 Ley B, Ryerson CJ, Vittinghoff E, et al. A multidimensional index and staging system for idiopathic pulmonary fibrosis. Ann Intern Med 2012; 156: 684-691.

10 Travis WD, Costabel U, Hansell DM, et al. An official American Thoracic Society/European Respiratory Society statement: Update of the international multidisciplinary classification of the idiopathic interstitial pneumonias. Am J Respir Crit Care Med 2013; 188: 733-748.

11 King TE Jr, Albera C, Bradford WZ, et al. Effect of interferon gamma-1b on survival in patients with idiopathic pulmonary fibrosis (INSPIRE): a multicentre, randomised, placebo-controlled trial. Lancet 2009; 374: 222-228.

12 du Bois RM, Weycker D, Albera C, et al. Ascertainment of individual risk of mortality for patients with idiopathic pulmonary fibrosis. Am J Respir Crit Care Med 2011; 184: 459-466.

13 Foster NR, Qi Y, Shi Q, et al. Tumor response and progression-free survival as potential surrogate endpoints for overall survival in extensive stage small-cell lung cancer: findings on the basis of North Central Cancer Treatment Group trials. Cancer 2011; 117: 1262-1271.

$14 \mathrm{Li} \mathrm{X}$, Liu S, Gu H, et al. Surrogate end points for survival in the target treatment of advanced non-small-cell lung cancer with gefitinib or erlotinib. J Cancer Res Clin Oncol 2012; 138: 1963-1969.

15 Fleming TR, Rothmann MD, Lu HL. Issues in progression-free survival when evaluating oncology products. J Clin Oncol 2009; 27: 2874-2880. 